\title{
Study of Glypican-3 in Patients with Hepatitis C Virus-Related Hepatocellular Carcinoma
}

\author{
ASHRAF G. DALA, M.D.*; EL-SAYED S. ABO EL-NOUR, M.D.**; \\ ABD ALLAH H. ARAKEEB, M.Sc.*** and AHMED EZZ EL-ARAB ABD EL-ALIM, M.D.* \\ The Departments of Internal Medicine* and Medical Biochemistry \& Molecular Biology**, Faculty of Medicine, \\ Menoufia University and The Department of Hepatology \& Gastroenterology, Shebin El-Kom Teaching Hospital ***, \\ Menoufia, Egypt
}

\begin{abstract}
Background: Early diagnosis and treatment of HCC can greatly improve the efficiency of treatment and extend patient life.

Aim of Study: To investigate the expression of serum Glypican-3 (GPC3) in patients with Hepatocellular Carcinoma (HCC) and determine its efficacy as a screening test in early detection of HCC.

Patients and Methods: This case control study involved $30 \mathrm{HCC}$ patients, 30 liver cirrhotic patients and 20 healthy controls. This study had been approved by local institutional research board in Menoufia Faculty of Medicine. All subjects participated in the study voluntarily and written informed consent was obtained from each participant. Clinical examination, abdominal ultrasonography and triphasic Computed Tomography (CT) for focal lesion were performed. Liver function tests were performed using clinical auto-analyzer, serum a-Fetoprotein (AFP) was measured using Enzymelinked Immune-Sorbent Assay (ELISA) method and GPC3 was determined by ELISA kit for GPC3. Data were collected and statistically analyzed.
\end{abstract}

Results: GPC3 was highly significant higher in HCC group than cirrhotic and control groups. There was highly positive significant correlation between GPC 3 and child score, size of focal lesions and number of focal lesions. The sensitivity of GPC 3 in diagnosis of HCC was $(68.5 \%)$ and the specificity was $(83.3 \%)$ at cut off point $(58.2 \mathrm{ng} / \mathrm{ml})$ that elicited from the Receiver Operator Characteristic (ROC) curve with very good Area Under Curve (AUC) (0.814), whereas that the sensitivity of AFP was (66.7\%) and the specificity was $(66.7 \%)$ at cut off point $(380 \mathrm{ng} / \mathrm{ml})$ that elicited from the ROC curve with very good AUC (0.679).

Conclusion: GPC 3 is highly associated with HCC and is more sensitive than AFP for early detection of HCC.

Key Words: Alpha-fetoprotein-Enzyme-linked immunesorbent assay - Glypican-3 - Hepatocellular carcinoma.

Correspondence to: Dr. Abd Allah H. Arakeeb, E-Mail: newpage77@yahoo.com

\section{Introduction}

HCC is the dominant variety of liver cancer; it represents the fourth leading cause of cancer deaths worldwide [1]. It constitutes about $70 \%$ of all liver tumors among Egyptians. The Hepatitis C Virus (HCV) infection is the most common risk factor of HCC in Egypt which leads to cirrhosis and severe liver damage [2]. About $60-80 \%$ of $\mathrm{HCV}$ infection leads to chronic hepatitis in the patients, and $10-20 \%$ of those patients develop cirrhosis within $20-30$ years. About 1-5\% of patients with cirrhotic liver might develop HCC [3]. Abdominal ultrasonography is well established to be used with or without AFP every 6 months as the standard surveillance strategy [4]. Some tumors do not produce AFP while others produce it with high levels. So, the diagnostic levels of AFP is variable and not the same in many studies [5], however, it is accepted now that levels of more than $20 \mathrm{ng} / \mathrm{ml}$ are diagnostic for HCC [6]. Glypican-3 (GPC3) is an oncofetal proteoglycan. It is attached to the hepatocyte cell wall. It is normally found in the embryonic hepatocyte but not in the normal mature liver. GPC3 is responsible of regulation, activation and depletion of various growth factors. This control depends on the power of glypicans to activate or suppress these growth factors and the reactions with their receptors [7]. A lot of studies have reported the involvement of GPC3 in many types of tumors, including HCC [8]. There is increasing evidence indicating that approximately $40 \%$ of HCC patients are positive for GPC 3 and negative for AFP [9]. The aim of this study is to investigate the expression of serum GPC 3 in patients with $\mathrm{HCC}$ and determine its efficacy as a screening test in early detection of HCC. 


\section{Patients and Methods}

This case control study consisted of 80 subjects who visited the Oncology and Internal Medicine Department at Menoufia Liver Institute during the period from June 2019 till December 2019.

This study had been approved by Local Institutional Research board in Menoufia Faculty of Medicine. All subjects participated in the study voluntarily and written informed consent was obtained from each participant. Subjects were classified into three groups, group I included 30 HCC patients, group II included 30 liver cirrhosis patients and group III included 20 healthy subjects as control group.

Inclusion criteria included patients with $\mathrm{HCV}$ related liver cirrhosis and $\mathrm{HCC}$, while patients with other malignancies or HCC distant metastasis were excluded.

Medical history taking and complete physical examination with particular emphasis on signs of chronic liver disease were done for all subjects.

Laboratory data included; Complete blood picture (CBC) analyzed in (an automated ADVIA120 hematological analyzer), data on liver function tests (AST, ALT, serum bilirubin, International Normalized Ratio (INR) and serum albumin) analyzed in (AU480 BECK Man, USA analyzer), serum AFP was measured using ELISA method and GPC 3 was determined by ELISA kit provided by (Chongqing Biospes Company, China), according to the recommendation of the manufacturer. The kit uses a double-antibody sandwich ELISA to assay the level of GPC3 samples.

Data were collected and statistically analyzed.

Radiological investigations were done like ultrasound and Triphasic CT on the abdomen and pelvis.

Clinical and Laboratory data of the cases were tabulated.

The study complied with the Faculty of Medicine, Menoufia University.

\section{Statistical analysis:}

The collected data was revised, coded and tabulated using Statistical package for Social Science (IBM Corp. Released 2017. IBM SPSS Statistics for Windows, Version 25.0 Armonk, NY: IBM Corp.). Data were presented and suitable analysis was done according to the type of data obtained for each parameter.
Mann Whitney Test (U-test) was done to test the normality of data distribution. Significant data was considered to be non parametric. Description of quantitative variables was in the form of mean and Standard Deviation (mean \pm SD), description of qualitative variables was by frequency and percentage, chi square test was used to assess the relationship between two qualitative groups. Fisher's exact test was used to examine the relationship between two qualitative variables when the expected count is less than 5 in more than $20 \%$ of cells. Correlation analysis was used to assess the strength of association between two quantitative variables; Spearman's correlation coefficient defines the strength and direction of the linear relationship between two variables. $p$-value 0.05 was set to be statistically significant and $p$-value 0.001 was set to be highly significant.

\section{Results}

Regarding age, the mean was $(57 \pm 7.3)$ years in HCC group, (53.7 \pm 6.6$)$ years in Cirrhotic group and (51.3 \pm 7.3$)$ years in Control group. So, there was no significant difference between $\mathrm{HCC}$ and cirrhotic groups ( $p_{1}$-value 0.07 ). Also there was no significant difference between HCC group and control group ( $p_{2}$-value 0.06$)$ and there was no significant difference between cirrhotic group and control group ( $p 3$-value 0.08 ). Regarding sex, HCC is more presented in males than in females. In HCC group there were 20 (66.7\%) males and 10 (33.3\%) females, in cirrhotic group there were 16 $(53.3 \%)$ males and $14(46.7 \%)$ females and in control group there were $13(65 \%)$ males and 7 $(35 \%)$ females. So, there was no significant difference among studied groups regarding sex ( $p$-value more than 0.05 for each) (Table 1).

Regarding laboratory tests, the mean White Blood Cells (WBCs) count was $\left(9.8 \pm 3.2 \times 10^{3}\right.$ / $\left.\mathrm{mm}^{3}\right),\left(6.7 \pm 2.3 \times 10^{3} / \mathrm{mm}^{3}\right)$ and $(8.7 \pm 1.4 \mathrm{X}$ $10^{3} / \mathrm{mm}^{3}$ ) for HCC, cirrhotic and control group respectively. So, there was no significant difference between studied groups regarding WBCs count ( $p$ value 0.133 ). Regarding platelets, the mean platelets count was $\left(105.1 \pm 32.9 \times 10^{3} / \mathrm{mm}^{3}\right),(93.60 \pm 30.6$ $\left.\mathrm{X} 10^{3} / \mathrm{mm}^{3}\right)$ and $\left(280.3 \pm 35.6 \times 10^{3} / \mathrm{mm}^{3}\right)$ for $\mathrm{HCC}$, cirrhotic and control group respectively. So, the mean platelets count was highly significant lower in cirrhotic group than control group ( $p_{1}$. value 0.001 ). The mean platelets count in HCC group was highly significant lower than control group ( $p_{2}$-value 0.001$)$. There was no significant difference between HCC and cirrhotic groups regarding platelets count ( $p_{3}$-value 0.194$)$. Regarding hemoglobin (Hgb) level, the mean Hgb level was 
$(10.8 \pm 1.2 \mathrm{gm} / \mathrm{dl}),(10.1 \pm 1.6 \mathrm{gm} / \mathrm{dl})$ and $(12.4 \pm 0.8$ $\mathrm{gm} / \mathrm{dl}$ ) for HCC, cirrhotic and control groups respectively. So, the mean Hgb level in cirrhotic group was highly significant lower than control group ( $p_{1}$-value 0.001$)$. The mean $\mathrm{Hgb}$ level in HCC was highly significant lower than control group $\left(p_{2}\right.$-value 0.001$)$. There was no significant difference between HCC and cirrhotic groups regarding $\mathrm{Hgb}$ level ( $p 3$-value 0.140). Regarding ALT, mean ALT level was $(60.3 \pm 19.3 \mathrm{u} / \mathrm{l}),(55.5 \pm$ $15.9 \mathrm{u} / \mathrm{l})$ and $(21.5 \pm 4.2 \mathrm{u} / \mathrm{l})$ for $\mathrm{HCC}$, cirrhotic and control group respectively. So, the mean ALT level in cirrhotic group was highly significant higher than control group ( $p_{1}$-value 0.001 ), and the mean ALT level in HCC group was highly significant higher than control group ( $p_{2}$-value 0.001$)$ with no significant difference between HCC and cirrhotic groups regarding mean ALT level ( $p_{3}$-value 0.394). Regarding AST, the mean AST value was (79.4 \pm $26.8 \mathrm{u} / \mathrm{l}),(71.5 \pm 21.2 \mathrm{u} / \mathrm{l})$ and $(23.6 \pm 4.2 \mathrm{u} / \mathrm{l})$ for $\mathrm{HCC}$, cirrhotic and control group respectively. So, the mean AST level in cirrhotic group was highly significant higher than control group ( $p_{1}$-value 0.001 ), and the mean AST level in HCC group was highly significant higher than control group ( $p 2-$ value 0.001 ) with no significant difference between HCC and cirrhotic groups regarding mean AST level ( $p 3$-value 0.527). Regarding Albumin, the mean Albumin level was $(3.0 \pm 0.4 \mathrm{~g} / \mathrm{dl}),(2.9 \pm 0.3$ $\mathrm{g} / \mathrm{dl})$ and $(4.0 \pm 0.2 \mathrm{~g} / \mathrm{dl})$ for $\mathrm{HCC}$, cirrhotic and control group respectively. So, the mean Albumin level in cirrhotic group was highly significant lower than control group ( $p_{1}$-value 0.001$)$. And the mean Albumin level in HCC group was highly significant lower than control group ( $p_{2}$-value 0.001 ). While, there was no significant difference between $\mathrm{HCC}$ and cirrhotic groups regarding mean Albumin level ( $p 3$-value 0.297). Regarding total bilirubin, the mean total bilirubin level was $(3.1 \pm 1.0$ $\mathrm{mg} / \mathrm{dl}),(1.6 \pm 0.5 \mathrm{mg} / \mathrm{dl})$ and $(0.9 \pm 0.2 \mathrm{mg} / \mathrm{dl})$ for $\mathrm{HCC}$, cirrhotic and control group respectively. So, there was no significant difference between cirrhotic and control groups regarding mean total bilirubin ( $p_{1}$-value 0.053$)$. While the mean total bilirubin level in HCC group was significant higher than control group ( $p_{2}$-value 0.001$)$ and the mean total bilirubin level in HCC group was significant higher than cirrhotic groups ( $p^{3}$-value 0.001). Regarding direct bilirubin, the mean direct bilirubin level was $(1.7 \pm 0.5 \mathrm{mg} / \mathrm{dl}),(0.6 \pm 0.2 \mathrm{mg} / \mathrm{dl})$ and $(0.2 \pm$ $0.1 \mathrm{mg} / \mathrm{dl}$ ) for HCC, cirrhotic and control group respectively. So, there was no significant difference between cirrhotic and control groups regarding mean direct bilirubin ( $p_{1}$-value 0.082$)$. While the mean direct bilirubin level in HCC group was significant higher than control group ( $p_{2}$-value
0.001), and the mean direct bilirubin level in HCC group was significant higher than cirrhotic groups ( $p 3$-value 0.001). Regarding INR, the mean INR value was $(1.5 \pm 0.3),(1.3 \pm 0.2)$ and $(1.0 \pm 0.2)$ for $\mathrm{HCC}$, cirrhotic and control group respectively. So, there was no significant difference between cirrhotic and control groups regarding mean INR value ( $p_{1}$-value 0.465$)$. While the mean INR value in HCC group was highly significant higher than control group ( $p_{2}$-value 0.001$)$. And, there was no significant difference between cirrhotic and HCC groups regarding mean INR value ( $p_{3}$-value 0.176$)$ (Table 2). Regarding AFP, mean AFP level was $(451.5 \pm 97.3 \mathrm{ng} / \mathrm{ml}),(10.7 \pm 23.9 \mathrm{ng} / \mathrm{ml})$ and $(3.2 \pm$ $1.6 \mathrm{ng} / \mathrm{ml}$ ) in HCC, cirrhotic and control groups respectively. So, the mean AFP level in cirrhotic group was highly significant higher than control group ( $p_{1}$-value 0.001$)$ and the mean AFP level in HCC group was highly significant higher than control group $\left(p_{2}\right.$-value 0.001$)$. And the mean AFP level in HCC group was significant higher than cirrhotic group ( $p_{3}$-value 0.017$)$. Regarding GPC3, the mean GPC3 level was $(25.3 \pm 28.6 \mathrm{~g} / \mathrm{L} / 6.1 \pm$ $10.9 \mathrm{~g} / \mathrm{L}$ and $(0.8 \pm 0.9 \mathrm{~g} / \mathrm{L}$ in HCC, cirrhotic and control groups respectively. So, there was no significant difference between cirrhotic and control groups regarding serum GPC3 level ( $p_{1}$-value 0.083 ) while the mean GPC3 level in HCC group was highly significant higher than control group $\left(p_{2}\right.$-value 0.001$)$. Also the mean GPC3 level in HCC group was highly significant higher than cirrhotic group ( $p 3$-value 0.001) (Table 3). For GPC 3 and other parameters in HCC group, our study showed that there was highly positive significant correlation between GPC 3 and child score with ( $p$-value 0.029$)$, size of focal lesions with ( $p$ value 0.011$)$ and number of focal lesions with ( $p$ value 0.001 ) in HCC group. There was negative significant between GPC3 and Albumin in HCC group with ( $p$-value 0.676 ), but there was negative non significant correlation between GPC 3 and age ( $p$-value 0.065$), \mathrm{WBCs}$ ( $p$-value 0.380$)$, Platelets ( $p$-value 0.927), Hgb ( $p$-value 0.470), ALT ( $p$ value 0.793$)$, AST ( $p$-value 0.907$)$, total bilirubin ( $p$-value 0.208$)$, direct bilirubin ( $p$-value 0.564$)$, INR ( $p$-value 0.462$)$, urea ( $p$-value 0.334$)$, creatinine ( $p$-value 0.914$)$ and AFP ( $p$-value 0.259$)$ in HCC group (Table 4). For AFP, AFP showed excellent poor $(\mathrm{AUC}=0.679)$. At cut off value of $380 \mathrm{ng} / \mathrm{mL}$, sensitivity was $66.7 \%$, specificity was $66.7 \%$, PPV was $66.7 \%$, NPV was $66.7 \%$, and accuracy was $66.7 \%$. For GPC3, GPC3 showed good AUC (AUC=0.814). At cut off value of 58.2 y/Lensitivity was $68.5 \%$, specificity was $83.3 \%$, PPV was $80 \%$, NPV was $71.4 \%$, and accuracy was $75 \%$. For combination of 2 tests showed excellent 
AUC (AUC=0.922). At cutoff point 380ng/ml for AFP and 58.2 g/Lfor GPC3, sensitivity was $70 \%$, specificity was $86.7 \%$, PPV was $84 \%$, NPV was $74.3 \%$, and accuracy was $78.4 \%$ (Table 5).

Table (1): Statically comparison among studied groups regarding age and sex.

\begin{tabular}{|c|c|c|c|c|c|c|}
\hline & $\begin{array}{c}\text { Control } \\
\mathrm{N}=20\end{array}$ & $\begin{array}{c}\mathrm{LC} \\
\mathrm{N}=30\end{array}$ & $\begin{array}{c}\mathrm{HCC} \\
\mathrm{N}=30\end{array}$ & $\begin{array}{c}\text { Statistical } \\
\text { test }\end{array}$ & $\begin{array}{c}p- \\
\text { value }\end{array}$ & $\begin{array}{c}\text { Post } \\
\text { Hoc test }\end{array}$ \\
\hline $\begin{array}{l}\text { Age (years): } \\
\text { Mean } \pm \mathrm{SD}\end{array}$ & $51.3 \pm 7.3$ & $53.7 \pm 6.6$ & $57 \pm 7.3$ & $\mathrm{~F}=1.7$ & 0.190 & $\begin{array}{l}p_{1}=0.08 \\
p_{2}=0.06 \\
p_{3}=0.070\end{array}$ \\
\hline $\begin{array}{c}\text { Males: } \\
\mathrm{N} \\
\%\end{array}$ & $\begin{array}{l}13 \\
65 \%\end{array}$ & $\begin{array}{l}16 \\
53.3 \%\end{array}$ & $\begin{array}{l}20 \\
66.7 \%\end{array}$ & $\chi^{2}=1.3$ & 0.527 & $\begin{array}{l}p_{1}=0.413 \\
p_{2}=0.903 \\
p_{3}=0.292\end{array}$ \\
\hline $\begin{array}{l}\mathrm{N} \\
\%\end{array}$ & $\begin{array}{l}7 \\
35 \%\end{array}$ & $\begin{array}{l}14 \\
46.7 \%\end{array}$ & $\begin{array}{l}10 \\
33.3 \%\end{array}$ & & & \\
\hline $\begin{array}{l}\text { HCC : Hepatoce } \\
\text { LC : Liver Cir } \\
\text { SD : Standard } \\
p \text {-value is signif } \\
p \quad: \text { Compari }\end{array}$ & ation. & & \multicolumn{4}{|c|}{$\begin{array}{l}p_{1}: \text { Comparison of LC versus control. } \\
p_{2}: \text { Comparison of HCC versus control. } \\
p_{3}: \text { Comparison between LC and HCC. } \\
\mathrm{F}: \text { Fisher's exact test. } \\
\chi^{2}: \text { Chi-square test. }\end{array}$} \\
\hline
\end{tabular}

Table (2): Statically comparison among studied groups regarding laboratory tests.

\begin{tabular}{|c|c|c|c|c|c|c|c|}
\hline Laboratory tests & Range & $\begin{array}{l}\text { Control } \\
\mathrm{N}=20\end{array}$ & $\begin{array}{c}\mathrm{LC} \\
\mathrm{N}=30\end{array}$ & $\begin{array}{l}\mathrm{HCC} \\
\mathrm{N}=30\end{array}$ & $\begin{array}{l}\text { Statistical } \\
\text { test }\end{array}$ & $\begin{array}{c}p- \\
\text { value }\end{array}$ & $\begin{array}{c}\text { Post } \\
\text { Hoc test }\end{array}$ \\
\hline $\begin{array}{l}W B C s\left(X 10^{9} / L\right): \\
\text { Mean } \pm \text { SD }\end{array}$ & $4000-11000$ & $8.7 \pm 1.4$ & $6.7 \pm 2.3$ & $9.8 \pm 3.2$ & $\mathrm{~F}=2.1$ & 0.133 & $\begin{array}{l}p_{1}=0.793 \\
p_{2}=0.106 \\
p_{3}=0.173\end{array}$ \\
\hline $\begin{array}{l}\text { Platelets }\left(\text { X } 10^{9} / \mathrm{L}\right) \text { : } \\
\quad \text { Mean } \pm \mathrm{SD}\end{array}$ & $150,000-4500^{\prime} 000$ & $280.3 \pm 35.6$ & $93.6 \pm 30.6$ & $105.1 \pm 32.9$ & $\mathrm{~F}=214.1$ & $0.001 *$ & $\begin{array}{l}p_{1}=0.001 * \\
p_{2}=0.001 * \\
p_{3}=0.194\end{array}$ \\
\hline $\begin{array}{l}\text { Hemoglobin }(g / d L) \text { : } \\
\quad \text { Mean } \pm \text { SD }\end{array}$ & $\begin{array}{l}\text { M: } 13.5-17.5 \\
\text { F: } 12.0-15.5\end{array}$ & $12.4 \pm 0.8$ & $10.1 \pm 1.6$ & $10.8 \pm 1.2$ & $\mathrm{~F}=20.4$ & $0.001 *$ & $\begin{array}{l}p_{1}=0.001 * \\
p_{2}=0.001 * \\
p_{3}=0.140\end{array}$ \\
\hline $\begin{array}{l}A L T(U / L): \\
\quad \text { Mean } \pm \mathrm{SD}\end{array}$ & $7-56$ & $21.5 \pm 4.2$ & $55.5 \pm 15.9$ & $60.3 \pm 19.3$ & $\mathrm{~F}=21.1$ & $0.001 *$ & $\begin{array}{l}p_{1}=0.001 * \\
p_{2}=0.001 * \\
p_{3}=0.394\end{array}$ \\
\hline $\begin{array}{l}A S T(U / L): \\
\quad \text { Mean } \pm \mathrm{SD}\end{array}$ & $10-40$ & $23.6 \pm 4.2$ & $71.5 \pm 21.2$ & $79.4 \pm 26.8$ & $\mathrm{~F}=9.1$ & $0.001 *$ & $\begin{array}{l}p_{1}=0.001 * \\
p_{2}=0.001 * \\
p_{3}=0.527\end{array}$ \\
\hline $\begin{array}{l}\text { Albumin }(g / d L): \\
\quad \text { Mean } \pm \text { SD }\end{array}$ & $3.5-5.5$ & $4.0 \pm 0.2$ & $2.9 \pm 0.3$ & $3.0 \pm 0.4$ & $\mathrm{~F}=78.8$ & $0.001 *$ & $\begin{array}{l}p_{1}=0.001 * \\
p_{2}=0.001 * \\
p_{3}=0.297\end{array}$ \\
\hline $\begin{array}{l}\text { Total bilirubin }(m g / d L): \\
\quad \text { Mean } \pm \text { SD }\end{array}$ & $0.1-1.2$ & $0.9 \pm 0.2$ & $1.6 \pm 0.5$ & $3.1 \pm 1.0$ & $\mathrm{~F}=20.9$ & $0.001 *$ & $\begin{array}{l}p_{1}=0.053 \\
p_{2}=0.001^{*} \\
p_{3}=0.001^{*}\end{array}$ \\
\hline $\begin{array}{l}\text { Direct bilirubin }(m g / d L) \\
\quad \text { Mean } \pm \text { SD }\end{array}$ & Less than 0.3 & $0.2 \pm 0.1$ & $0.6 \pm 0.2$ & $1.7 \pm 0.5$ & $\mathrm{~F}=27.9$ & $0.001 *$ & $\begin{array}{l}p_{1}=0.082 \\
p_{2}=0.001 * \\
p_{3}=0.001 *\end{array}$ \\
\hline $\begin{array}{l}\text { INR: } \\
\quad \text { Mean } \pm \mathrm{SD}\end{array}$ & $0.8-1.2$ & $1.1 \pm 0.1$ & $1.3 \pm 0.2$ & $1.5 \pm 0.2$ & $\mathrm{~F}=36.2$ & 0.231 & $\begin{array}{l}p_{1}=0.465 \\
p_{2}=0.001 * \\
p_{3}=0.176\end{array}$ \\
\hline \multicolumn{2}{|c|}{$\begin{array}{l}\text { HCC }: \text { Hepatocellular-Carcinoma. } \\
\text { LC }: \text { Liver Cirrhosis. } \\
\text { SD : Standard Deviation. } \\
p \text {-value is significant if }<0.05 . \\
p \quad: \text { Comparison between control, LC and HCC }\end{array}$} & $\begin{array}{ll}p_{1}: \text { Comp } \\
p_{2}: \text { Comp } \\
p_{3}: \text { Comp } \\
\text { WBCs : White } \\
\text { ALT : Alani }\end{array}$ & $\begin{array}{l}\text { ison of LC ve } \\
\text { ison of HCC } \\
\text { ison between } \\
\text { lood Cells. } \\
\text { Transaminas }\end{array}$ & $\begin{array}{l}\text { us control. } \\
\text { rsus control. } \\
\text { C and HCC. } \\
\text { Enzyme. }\end{array}$ & \multicolumn{3}{|c|}{$\begin{array}{l}\text { AST: Aspartate Transaminase Enzyme. } \\
\text { INR : International Normalized Ratio. } \\
\mathrm{F} \quad: \text { Fisher's exact test. } \\
{ }^{*} \\
\mathrm{~N}\end{array}$} \\
\hline
\end{tabular}


Table (3): Statically comparison among studied groups regarding AFP and GPC3 test.

\begin{tabular}{|c|c|c|c|c|c|c|c|c|}
\hline & Norma range & & $\begin{array}{c}\text { Control } \\
\mathrm{N}=20\end{array}$ & $\begin{array}{c}\mathrm{LC} \\
\mathrm{N}=30\end{array}$ & $\begin{array}{l}\mathrm{HCC} \\
\mathrm{N}=30\end{array}$ & $\begin{array}{l}\text { Statistical } \\
\text { test }\end{array}$ & $\begin{array}{c}p- \\
\text { value }\end{array}$ & $\begin{array}{c}\text { Post } \\
\text { Hoc test }\end{array}$ \\
\hline$A F P(n g / m L):$ & Less than 10: & $\begin{array}{l}\text { Mean } \\
\text { SD } \\
\text { Median } \\
\text { Min } \\
\text { Max }\end{array}$ & $\begin{array}{l}3.2 \\
1.6 \\
3.5 \\
1 \\
7\end{array}$ & $\begin{array}{l}10.7 \\
23.9 \\
5 \\
1 \\
108\end{array}$ & $\begin{array}{l}451.5 \\
97.3 \\
452.5 \\
133.2 \\
663\end{array}$ & $\mathrm{~K}=28.9$ & $0.001 *$ & $\begin{array}{l}p_{1}=0.001 * \\
p_{2}=0.001 * \\
p_{3}=0.17\end{array}$ \\
\hline Glypican-3 ( $\mathrm{g} / \mathrm{h})$ : & $0.7-15$ & $\begin{array}{l}\text { Mean } \\
\text { SD } \\
\text { Median } \\
\text { Min } \\
\text { Max }\end{array}$ & $\begin{array}{l}0.8 \\
0.9 \\
0.4 \\
0.03 \\
3.6\end{array}$ & $\begin{array}{l}6.1 \\
10.9 \\
1 \\
0.1 \\
48\end{array}$ & $\begin{array}{l}25.3 \\
28.6 \\
17.9 \\
0.9 \\
93.3\end{array}$ & $K=36.2$ & $0.001 *$ & $\begin{array}{l}p_{1}=0.083 \\
p_{2}=0.001 * \\
p_{3}=0.001 *\end{array}$ \\
\hline $\begin{array}{l}\text { HCC : Hepatocellular } \\
\text { LC : Liver Cirrhosis } \\
\text { AFP : Alfa Feto-Prote } \\
\text { SD : Standard Devia }\end{array}$ & on. & $\begin{array}{l}\text { : Compari } \\
\text { : Compari } \\
\text { : Compari }\end{array}$ & $\begin{array}{l}\text { n between } \\
\text { n of LC ve } \\
\text { n of HCC }\end{array}$ & $\begin{array}{l}\text { ontrol, L } \\
\text { sus contr } \\
\text { ersus cor }\end{array}$ & $\begin{array}{l}\mathrm{C} \text { and } \mathrm{H} \\
\text { l. } \\
\text { irol. }\end{array}$ & $\begin{aligned} \mathrm{K}_{3} & : \mathrm{Cc} \\
& : \mathrm{K} \\
* & : \mathrm{Si} \\
\mathrm{N} & : \mathrm{N}\end{aligned}$ & $\begin{array}{l}\text { skal-Wal } \\
\text { hificant. } \\
\text { aber. }\end{array}$ & $\begin{array}{l}\text { tween LC } \\
\text { test. }\end{array}$ \\
\hline
\end{tabular}

Table (4): Statically comparison between glypican-3 and other parameters in $\mathrm{HCC}$ group.

\begin{tabular}{lcc}
\hline & & Glypican-3 \\
Parameters & $r$ & $p$ \\
\cline { 2 - 3 } & & 0.341 \\
\hline Age & -0.166 & 0.065 \\
WBCs & -0.018 & 0.380 \\
Platelets & -0.137 & 0.927 \\
Hemoglobin & -0.050 & 0.470 \\
ALT & -0.022 & 0.793 \\
AST & 0.080 & 0.907 \\
Albumin & -0.237 & 0.676 \\
Total bilirubin & -0.110 & 0.208 \\
Direct bilirubin & 0.139 & 0.564 \\
INR & 0.183 & 0.462 \\
Urea & 0.213 & 0.334 \\
Creatinine & 0.020 & 0.259 \\
AFP & 0.399 & 0.914 \\
CHILD score & 0.703 & 0.029 \\
Size of lesions & 0.819 & 0.011 \\
Number of lesions & & \\
\hline$r$ & & \\
WBCs : Spearman's correlation coefficient. & \\
ALT : Alanine Transaminase Enzyme. & \\
AST : Aspartate Transaminase Enzyme. & \\
INR : International Normalized Ratio. & \\
AFP : Alpha-Fetoprotein. & & \\
HCC : Hepatocellular-Carcinoma. & \\
$p$ & & \\
& & \\
\end{tabular}

Table (5): Sensitivity and specificity of AFP and GPC3 for screening of HCC.

\begin{tabular}{llcc}
\hline & AFP & Glypican-3 & $\begin{array}{c}\text { Combination of } \\
\text { Glypican-3 and AFP }\end{array}$ \\
\hline AUC & 0.679 & 0.814 & 0.856 \\
Cut off & 380 & 58.2 & 70 \\
Sensitivity (\%) & 66.7 & 68.5 & 86.7 \\
Specificity (\%) & 66.7 & 83.3 & 84 \\
PPV (\%) & 66.7 & 80 & 74.3 \\
NPV (\%) & 66.7 & 71.4 & 78.4 \\
Accuracy (\%) & 66.7 & 75 & \\
\hline
\end{tabular}

AUC : Area Under Curve. $\quad$ PPV : Positive Predictive Value. ROC : Receiver Operating Curve. NPV : Negative Predictive Value Rough AUC Guidelines: The area under the ROC curve (AUC) results were considered excellent for AUC values between 0.9-1, good for AUC values between 0.8-0.9, fair for AUC values between 0.7-0.8, poor for AUC values between 0.6-0.7 and failed for AUC values between 0.5-0.6).

\section{Discussion}

In the current study, the mean age was $(57 \pm 7.3)$ years in HCC group, $(53.7 \pm 6.6)$ years in cirrhotic group and (51.3 \pm 7.3$)$ years in control group. So, there was no significant difference between HCC and cirrhotic groups. Also there was no significant difference between HCC group and control group and there was no significant difference between cirrhotic group and control group. Our study in agreement with Omar et al., [10] who reported that the mean age was 56.9 \pm 7.27 years in HCC group while was $52.89 \pm 9.24$ years in cirrhotic group. Our results showed that HCC was highly prevalent in males, there were $20(66.7 \%)$ males and 10 (33.3\%) females in HCC group, in cirrhotic group there were $16(53.3 \%)$ males and $14(46.7 \%)$ females and in control group there were $13(65 \%)$ males and 7 (35\%) females. So, there was no significant difference among studied groups regarding sex. Our results agreed with Badr et al., [2] who reported that in $\mathrm{HCC}$ group they were 25 males and 5 females, in cirrhotic group they were 23 males and 7 females. Our study showed regarding platelets count the mean platelets count was highly significant lower in cirrhotic group than control group but there was no significant difference between HCC and cirrhotic groups regarding platelets count and regarding $\mathrm{Hgb}$ concentration showed no significant difference between HCC and cirrhotic group and highly significant difference between HCC and cirrhotic groups and control group being lower $\mathrm{Hgb}$ concentration in cirrhotic and HCC groups. Our results were agreed with Omar et al., [10] who showed that the mean $\mathrm{Hgb}$ concentration was $(9.37 \pm 1.57 \mathrm{gm} / \mathrm{dl})$ in cirrhotic group while in $\mathrm{HCC}$ group it was $(9.98 \pm 1.28 \mathrm{gm} / \mathrm{dl})$ with no significant difference regarding the mean $\mathrm{Hgb}$ concentration. Also, the platelets count show no significant difference between HCC group (131.38 \pm 
$\left.74.710^{3} \mathrm{X} \mathrm{mm}^{3}\right)$ and cirrhotic group $(98.66 \pm 61.09$ $10^{3} \mathrm{X} \mathrm{mm}^{3}$ ). In contrary to our results, ParanaguáVezozzo et al., [11] who showed that the mean Platelets count was significant lower in HCC group $\left(83.910^{3} \mathrm{X} \mathrm{mm}^{3}\right)$ than cirrhotic group (118.5 $10^{3}$ $\mathrm{X} \mathrm{\textrm {mm } ^ { 3 }}$ ), the lower blood platelet count in HCC patients can be explained by a longer evolution of chronic liver disease with subsequent advanced portal hypertension and hypersplenism. Our study showed that the mean ALT and AST value were highly significant higher in HCC group and cirrhotic group than in control group with no significant difference between HCC group and cirrhotic group. Our study showed that there was highly significant difference regarding mean values of serum albumin, total and direct bilirubin and INR between HCC group and control group being lower albumin, higher total, direct bilirubin and INR in HCC group, also showed no significant difference regarding serum albumin, total bilirubin direct bilirubin and INR between HCC and cirrhotic groups. Also there was highly significant difference regarding mean values of serum albumin, but there was no significant difference regarding total, direct bilirubin and INR between cirrhotic and control groups. Our results agreed with Omar et al., [10] who showed that both ALT and AST mean values were highly significant higher in HCC group $(59.4 \pm 37.1 \mathrm{u} / \mathrm{l}$ for ALT and $85.4 \pm 65.8 \mathrm{u} / \mathrm{l}$ for AST) and cirrhotic group $(49.28 \pm 26.7 \mathrm{u} / \mathrm{l}$ for ALT and $66.83 \pm 42.2 \mathrm{u} / \mathrm{l}$ for AST) than in control group $(23.77 \pm 6.5 \mathrm{u} / \mathrm{l}$ for ALT and $24.07 \pm 6.9 \mathrm{u} / \mathrm{l}$ for AST), while there was no significant difference between HCC group and cirrhotic group, also there were statistically highly significant low mean values of serum albumin in both HCC and cirrhotic groups being $2.24+0.48 \mathrm{gm} / \mathrm{dl}$ and $2.7+0.54 \mathrm{gm} / \mathrm{dl}$ respectively. Our results were in contrary to ParanaguáVezozzo et al., [11] who showed that both ALT and AST mean values were significant higher in HCC group (70u/l for ALT and $91 \mathrm{u} / \mathrm{l}$ for AST) than in cirrhotic group (47u/l for ALT and 53u/l for AST). Our study showed that there was highly positive significant correlation between GPC 3 and child score, size of focal lesions and number of focal lesions in HCC group. There was negative significant between GPC3 and Albumin in HCC group. But there was negative non significant correlation between GPC3 and age, WBCs, Platelets, Hgb, ALT, AST, total bilirubin, direct bilirubin, INR, and AFP in HCC group. Our study comes in agreement with Jia et al., [12] who reported that there was no significant correlation between serum levels of AFP and GPC3. Also Jia et al., [12] reported that there was no statistical association between serum GPC3 and age, gender, Child-Pugh score, AFP level, number of tumors, or tumor size. In contrast to our study Mohamed [13] reported that there was no significant association between tumor size and the level of GPC3 and the level of AFP. Although the test for AFP is widely available, inexpensive, and easy to perform, it has poor accuracy as a serological test for the early detection of HCC. Levels of AFP increase not only in people with HCC, but also in people with active hepatitis, cirrhosis without HCC, or exacerbation of the underlying liver disease, due to pathophysiological changes of inflammation and regeneration; this means the test can have low specificity in the population at risk Gopal et al., [14] . Our study showed that the mean AFP level in HCC group was significant higher than cirrhotic group while the mean AFP level in HCC group was highly significant higher than control group and the mean AFP level in cirrhotic group was highly significant higher than control group. Our results were close to Ismail et al., [15] who reported that AFP showed a significant elevation in the HCC group (4901.367 $\pm 2185.800 \mathrm{ng} / \mathrm{ml}$ ) compared to the control group $(4.033 \pm 1.191 \mathrm{ng} / \mathrm{ml})$ and cirrhotic liver group $(100.733 \pm 71.726 \mathrm{ng} / \mathrm{ml})$. Our study showed that the mean GPC3 level in HCC group was highly significant higher than cirrhotic and control groups and the mean GPC3 level in cirrhotic group was highly significant higher than control group. Also these results come in agreement with Yang et al., [16] who had demonstrated that serum GPC3 level was significantly higher in HCC patients than those in control group subjects, and patients with hepatitis or liver cirrhosis. Our study showed that AFP showed excellent poor (AUC=0.679). At cut off value of $380 \mathrm{ng} / \mathrm{mL}$, sensitivity was $66.7 \%$, specificity was $66.7 \%$, PPV was $66.7 \%$, NPV was $66.7 \%$, and accuracy was $66.7 \%$. Our results were close to also our results were close to Jia et al., [12] who reported that of the 102 samples from patients with HCC, 53 samples $(51.96 \%)$ were positive for AFP at cut off point $(400 \mathrm{ng} / \mathrm{ml})$. In our study GPC3 showed good AUC (AUC=0.814). At cut off value of $58.2 \mathrm{~g} / \mathrm{L}$ sensitivity was $68.5 \%$, specificity was $83.3 \%$, PPV was $80 \%$, NPV was $71.4 \%$, and accuracy was $75 \%$. Our result come in agreement with Mohamed [13] in which GPC3 was less than $2 \mathrm{ng} / \mathrm{ml}$ in sera of healthy subjects and patients with liver cirrhosis, but its level was significantly increased in $93.3 \%$ (28/30) of patients with HCC more than $4 \mathrm{ng} / \mathrm{ml}$. In addition, only 3 (10\%) patients with cirrhosis displayed elevated levels of serum GPC3 from $2-4 \mathrm{ng} / \mathrm{ml}$. At a cut off value of $2.72 \mathrm{ng} / \mathrm{ml}$, serum GPC 3 has sensitivity $(93.0 \%)$ and specificity (94\%). Our study according to the combination of AFP and GPC 3 tests, showed ex- 
cellent AUC (AUC=0.922). At cutoff point 380 $\mathrm{ng} / \mathrm{ml}$ for alpha-fetoprotein and $58.2 \mathrm{gg} / \mathrm{L}$ for GPC3, sensitivity was $70 \%$, specificity was $86.7 \%$, PPV was $84 \%$, NPV was $74.3 \%$, and accuracy was $78.4 \%$. This comes in agreement with the study of Sun et al., [8] in which the sensitivity and specificity of combined detection of AFP and GPC3 reached $85.5 \%$ and $91.5 \%$, respectively.

\section{Conclusion:}

We concluded that GPC 3 is a valuable serum marker that can aid the early diagnosis of HCC. In combination, measurements of AFP and GPC3 have the advantage to improve the detection of one of the most common malignancies worldwide.

\section{References}

1- EL-SERAG H.B.: Epidemiology of hepatocellular carcinoma. The Liver: Biology and Pathobiology, 758-72, 2020.

2- BADR E.A., KORAH T.E., GHANI A.A., EL-SAYED S and BADR S.: Role of serum glypican-3 in the diagnosis and differentiation of small hepatocellular carcinoma from hepatitis-C virus cirrhosis. Alexandria Journal of Medicine, 50 (3): 221-6, 2014.

3- GOMAA A., ALLAM N., ELSHARKWAY A., EL KASSAS M. and WAKED I.: Hepatitis C infection in Egypt: Prevalence, impact and management strategies. Hepatic medicine: Evidence and Research, 9, 17, 2017.

4- HEIMBACH J.K., KULIK L.M., FINN R.S., SIRLIN C.B., ABECASSIS M.M., ROBERTS L.R., ZHU A.X., MURAD M.H. and MARRERO J.A.: AASLD Guidelines for the Treatment of Hepatocellular Carcinoma. Hepatology, Vol. 67, No. 1, 2018.

5- LLOVET J.M., VILLANEUVA A., LACHENMAYER A. and FINN R.S.: Advances in targeted therapies for hepatocellular carcinoma in the genomic era. Nat. Rev. Clin. Oncol., 12: 408-24, 2015.

6- SHABANA M.A., ABDELKADER A.H. and AFIFI S.A.A.: Evaluation of HCV-associated Hepatocellular Carcinoma based on Alpha-fetoprotein levels. AfroEgyptian Journal of Infectious and Endemic Diseases, 10 (1): 10-15, 2020.
7- FILMUS J. and CAPURRO M.: Glypican-3: A marker and a therapeutic target in hepatocellular carcinoma. FEBS J. May; 280 (10): 2471-6. Doi: 10.1111/febs. 12126. Epub. 2013 Jan. 31, 2013.

8- SUN B., HUANG Z., WANG B., YU Y., LIN S., LUO L. and HUANG Z.: Significance of glypican-3 (GPC3) expression in hepatocellular cancer diagnosis. Medical science monitor: International medical journal of experimental and clinical research, 23: 850, 2017.

9- YU J., XU X., MA R., et al.: Development of a clinical chemiluminescent immunoassay for serum GPC3 and simultaneous measurements alone with AFP and CK19 in diagnosis of hepatocellular carcinoma. Journal of clinical laboratory analysis, 29: 85-93, 2015.

10- OMAR S.A., HASAN B.B., GAMAL W. and HASAN A.A.: Is Glypican-3 useful Diagnostic Marker that Distinguishes Hepatocellular Carcinoma from Liver Cirrhosis? Afro-Egyptian Journal of Infectious and Endemic Diseases, 10 (1): 32-8, 2020

11- PARANAGUÁ-VEZOZZO D.C., MATIELO C.E.L., De CAMPOS D.F., De LIMA R., ZITELLI P.M.Y., ONO S.K. and CARRILHO F.J.: A potential clinical based score in hepatitis $\mathrm{C}$ virus cirrhotic patients to exclude small hepatocellular carcinoma, 2018.

12- JIA X.B., GAO Y.T., ZHAI D.K., LIU J., CAI J.J., WANG Y.J., JING L., et al.: Assessment of the Clinical Utility of Glypican 3 as a SerumMarker for the Diagnosis of Hepatocellular Carcinoma. Technol. Cancer Res. Treat., 15: 780-6, 2016.

13- MOHAMED M.S.: Value of serum Glypican 3 as a predictive tool for early diagnosis of hepatocellular carcinoma (HCC). Al-Azhar International Medical Journal, 2020.

14- GOPAL P., YOPP A.C., WALJEE A.K., CHIANG J., NEHRA M., KANDUNOORI P., et al.: Factors that affect accuracy of $\alpha$-fetoprotein test in detection of hepatocellular carcinoma in patients with cirrhosis. Clinical Gastroenterology and Hepatology, 12: 870-7, 2014.

15- ISMAIL S., EL SAADANY S., ZIADA D., et al.: Cytokeratin-18 in diagnosis of HCC in patients with liver cirrhosis. Asian Pac J. Cancer Prev., 18: 1105-11, 2017.

16- YANG S.L., FANG X., HUANG Z.Z., LIU X.J., XIONG Z.F., LIU P., YAO H.Y., et al.: Can serum glypican-3 be a biomarker for effective diagnosis of hepatocellular carcinoma? A meta-analysis of the literature. Dis. Markers, 2014: 127831, 2014. 


\section{دراسة جليبكان-r فى سرطان الكبد المرتبط

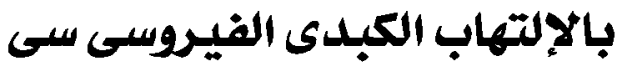

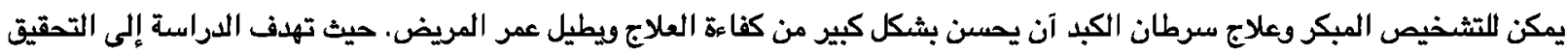
من تعير هصل جليكان-r (GPC3) فى مرضى سرطان الخلايا الكبدية وتحديد فعاليته كإختبار تحرى فى الكثف المبكر عن سرطان الخلايا الكبدية.

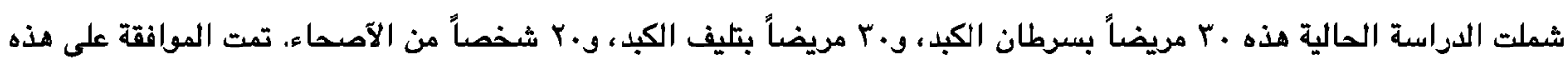

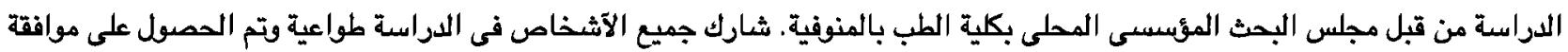

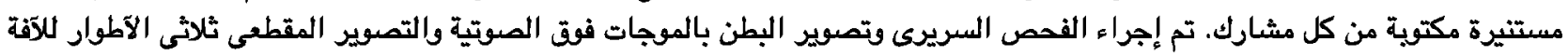

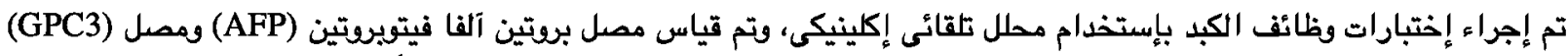

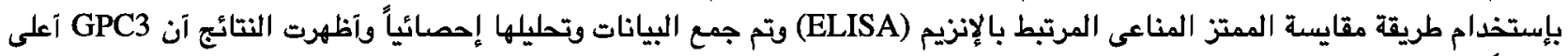

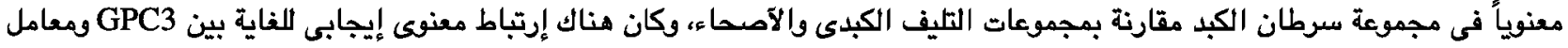

(CHILD) ) وحجم الآفات البؤرية وعدد الآفات البؤدية.

وخلمنا إلى آن مصل جليكان-بّ يرتبط إرتباطاً وثيقاً بسرطان الكبد وهو آكثر حساسية من مصل بروتين آلفا فيتوبريتين للكثف المبكر عن سرطان الكبد. 